\title{
The development of direct extrusion-injection moulded zein matrices as novel oral controlled drug delivery
}

\section{systems}

Jacob Bouman ${ }^{* *}$, Peter Belton ${ }^{\$}$, Paul Venema ${ }^{*}$, Erik van der Linden ${ }^{*}$, Renko de Vries ${ }^{\dagger \ell} \&$ Sheng

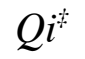

Laboratory of Physical Chemistry and Colloid Science, Wageningen University, Wageningen, The Netherlands.

*Laboratory of Physical chemistry and Physics of foods, Wageningen University, Wageningen, The Netherlands.

"School of Pharmacy, University of East Anglia, Norwich, United Kingdom.

\$ School of Chemistry, University of East Anglia, Norwich, United Kingdom

$£$ Department of Biomedical Engineering, University of Groningen and University Medical Centre Groningen, Groningen, The Netherlands

Corresponding author: Sheng Qi, sheng.qi@uea.ac.uk and Jacob Bouman, jacob.bouman@wur.nl 


\section{ABSTRACT}

Purpose To evaluate the potential of zein as a sole excipient for controlled release formulations prepared by hot melt extrusion.

Methods Physical mixtures of zein, water and crystalline paracetamol were hot melt extruded (HME) at $80^{\circ} \mathrm{C}$ and injection moulded (IM) into caplet forms. HME-IM Caplets were characterised using differential scanning calorimetry, ATR-FTIR spectroscopy, scanning electron microscopy and powder X-ray diffraction. Hydration and drug release kinetics of the caplets were investigated and fitted to a diffusion model.

Results For the formulations with lower drug loadings, the drug was found to be in the noncrystalline state, while for the ones with higher drug loadings paracetamol is mostly crystalline. Release was found to be largely independent of drug loading but strongly dependent upon device dimensions, and predominately governed by a Fickian diffusion mechanism, while the hydration kinetics shows the features of Case II diffusion.

Conclusions In this study a prototype controlled release caplet formulation using zein as the sole excipient was successfully prepared using direct HME-IM processing. The results demonstrated the unique advantage of the hot melt extruded zein formulations on the tuneability of drug release rate by alternating the device dimensions.

\section{KEY WORDS}

Zein, hot melt extrusion-injection moulding, controlled release, dissolution kinetics modelling, diffusion mechanism 


\section{ABBREVIATIONS}

HME

IM

HME-IM

$\operatorname{Tg}$

DSC

ATR-FTIR

PXRD

SEM

RH
Hot melt extrusion

Injection moulding

Hot melt extrusion-injection moulding

Glass transition temperature

Differential Scanning Calorimetry

Attenuated Total Reflection Fourier Transform Infrared

Spectroscopy

Powder X-ray diffraction

Scanning electron microscopy

Relative humidity 


\section{INTRODUCTION}

Controlled release oral drug delivery systems have many advantages over conventional formulations with no constraint on the drug release rate, including reduced dosing frequency, enhanced bioavailability and subsequently reduced side-effects by maintaining a long period of steady drug plasma concentration (1). Therefore the use of controlled release formulations often contributes to the improved patient adherence to the treatment and overall therapeutic outcome. However, good control of the drug release rate is challenging and polymeric excipients are often used for this purpose (2). Although there is a wide range of synthetic polymers may be used for controlled release purpose, some naturally derived polymers have the advantages of being biocompatible as well as environmentally sustainable and often inexpensive. Therefore, much interest has been directed towards finding more natural polymers as suitable excipients for drug delivery formulations including controlled drug release systems. Recently zein, a mixture of natural proteins found in maize, has been explored for its potential applications in drug delivery $(3-5)$.

Zein is a main component in the by-products of both the wet milling and dry milling process to obtain ethanol from maize (6). It contains a mixture of $\alpha, \beta, \gamma$ and $\delta$ zein proteins which contains an equal amounts of hydrophilic and hydrophobic amino acid residues, therefore it forms hydrophobic and hydrophilic domains allowing it to behave as a polymeric amphiphile (7). It is not readily soluble in water which is likely to be due to strong protein-protein interactions (8). Compared to other proteins, zein has good heat and pH stabilities (9). It is highly biocompatible $(10,11)$, low cost and has versatile physical and mechanical properties that are suitable for being used in many different forms of drug delivery systems. For example, zein has been already 
successfully applied in the preparation of drug-loaded microspheres (3), films (4) and tablets (5). In addition, the swellable but non-dissolvable nature of zein makes it as a suitable candidate of excipient for controlled release drug delivery systems.

Being relatively heat-stable, zein has proven to be a good candidate for hot melt extrusion $(12,13)$, which is a thermal processing that is being increasingly adopted for the preparation of pharmaceutical solid dispersion based formulations. It has been reported that zein's structure only begin to change at temperatures above $120^{\circ} \mathrm{C}$, which is likely to be due to protein cross linking by disulfide bonds (9). The use of extrusion techniques to process zein is well known in the food industry $(12,13)$, but the potential for the preparation of pharmaceutical products has not been widely explored. The aim of this work is to develop new oral controlled release caplets with tuneable release rates with zein as the sole excipient using a simple two-step processing, hot melt extrusion (HME) in combination with injection moulding (IM). The use of HME in combination with IM is a novel and effective way to create tuneable matrix dosage forms with precise shape and dimensions $(14,15)$, which is critical for the systems in which the drug release rate is highly controlled by the dimension and shape of the device.

During extrusion zein is heated above the glass transition temperature $\left(\mathrm{T}_{\mathrm{g}}\right)$ and forms a viscoelastic system (9) generating a single matrix phase providing potentially superior properties compared to those prepared by tablet pressing, where particles are only pressed together forming an inhomogeneous system. Paracetamol was used as a model drug because it is well characterised, $\mathrm{pH}$ stable and stable at the extrusion temperatures applied in this study (16). The physical states of the model drug and its distribution was characterised by differential scanning 
calorimetry (DSC), Fourier transform Infra-red spectroscopy (FTIR) and powder X-ray diffraction (PXRD). Dissolution studies were performed to investigate the influence of dissolution medium, drug loading and caplet dimension on the release kinetics. Hydration behaviour of the caplets was monitored and the potential impacts on drug release kinetics were evaluated.

\section{MATERIALS AND METHODS}

\section{Materials}

Purified zein protein (Acros organics, Geel, Belgium) was ground using a kitchen blender with 15 s grinding alternated with 20 s cooling down to minimise temperature increases due to frictional forces. The particle size of the grounded zein powder was measured between 40-200 $\mu \mathrm{m}$ to ensure good mixing with the crystalline model drug, paracetamol (Sigma-Aldrich, Gillingham, UK), which had similar particle size.

\section{Hot melt extrusion and injection moulding}

Ground zein power was mixed with distilled water (10-12\% of weight mixture) in a glass mortar and pestle in order to lower its glass transition temperature $\left(\mathrm{T}_{\mathrm{g}}\right)$ and allow the hot melt extrusion at relatively low temperature. Different amount of paracetamol was added to the zein-water mixture to obtain the mixes for formulations with drug loading ranging from $4.4-45.1 \%(\mathrm{w} / \mathrm{w})$. The ternary mixes (zein-water-model drug) were ground again prior to extrusion. A feeding batching size of 5-10g was processed using a HAAKE ${ }^{\mathrm{TM}}$ Minilab extruder (Thermo Fisher, Karlsruhe, Germany) with a co-rotating twin screw. The extrusions were performed at a 
temperature of $80^{\circ} \mathrm{C}$ and screw speed of $100 \mathrm{rpm}$. A pre-heated injection moulding cylinder ( 80 ${ }^{\circ} \mathrm{C}$ ) was attached to the extruder to collect the extrudates and keep them at $80^{\circ} \mathrm{C}$. The injection moulding process was performed at $80^{\circ} \mathrm{C}$, under high pressure of $300-500$ bar into a caplet mould using a HAAKE ${ }^{\mathrm{TM}}$ MiniJet Pro Piston Injection Moulding System (Thermo Fisher, Karlsruhe, Germany). After the IM sequence, the mould was placed on aluminium foil positioned on dry ice for a rapid cooling. The caplet $(5.9 \times 4.4 \times 19.7 \mathrm{~mm})$ was then ejected out of the mould and stored in a sealed container at ambient conditions. After processing, the moisture contents of the caplets were determined using thermogravimetric analysis (TGA) to be between $12 \%$ and $14 \%(\mathrm{w} / \mathrm{w})$ for the samples with drug loadings between $45.1 \%$ to $4.4 \%(\mathrm{w} / \mathrm{w})$.

\section{Differential Scanning Calorimetry (DSC)}

DSC experiments were conducted using a Q2000 MTDSC (TA Instruments, Newcastle, DE, U.S.). Full range calibration was performed prior to the sample testing. For the DSC samples, a caplet slice was cut and milled into smaller particles using mortar and pestle. Prior to the DSC measurements, all samples were dried in a $70^{\circ} \mathrm{C}$ oven for 3 days, to minimize the effect of water evaporation during a DSC run. A standard heat-cool-heat sequence was applied to each sample. $10.00{ }^{\circ} \mathrm{C} / \mathrm{min}$ heating rate and $5.00^{\circ} \mathrm{C} / \mathrm{min}$ cooling rate were applied for all samples. In the DSC cell a nitrogen purge at a flow rate of $50 \mathrm{~mL} / \mathrm{min}$ was used. Analysis was performed using TA Universal Analysis software. To measure the amount of crystalline paracetamol present, melting enthalpies were compared with the melting enthalpy of pure crystalline paracetamol form I, which was experimentally measured as $181 \pm 0.7 \mathrm{~J} / \mathrm{g}$.

\section{Attenuated Total Reflection Fourier Transform Infrared Spectroscopy (ATR-FTIR)}


ATR-FTIR spectra of the samples were collected using an FTIR spectrometer (IFS66/S model from Bruker Optics limited, Coventry, UK) with a mercury/cadmium/telluride detector. The samples (both outer surface and cross-section of caplet/extrudate) were directly placed on a single-reflection diamond ATR (attenuated total reflectance) accessory (Specac, Orpington, UK). 64 scans were acquired for each sample with a resolution of $2 \mathrm{~cm}^{-1}$.

\section{Powder X-Ray Diffraction (PXRD)}

PXRD measurements were taken using a Thermo Scientific ${ }^{\mathrm{TM}}$ ARL $^{\mathrm{TM}}$ X'TRA Powder $^{\prime}$ Diffractometer (Ecublens, Switzerland) fitted with a $\mathrm{Cu}$ (copper) x-ray tube. The following operating conditions were used: voltage, $45 \mathrm{kV}$; current, $40 \mathrm{~mA}$; step size, $0.01^{\circ}$ and acquisition time of 0.5-1.0 s/step.

\section{Scanning electron microscopy (SEM)}

Prior to SEM imaging, fractured caplet pieces were sputter coated with Au/Pd. The images of the morphologies of the surface and cross section of the caplets/extrudates were taken using a Phillips XL20 SEM (Phillips Electron Optics, Netherlands).

\section{Swelling and hydration studies}

The swelling behaviour of the formulations was investigated using an imaging based method. Several cylindrical pieces of drug-loaded zein extrudate with similar diameters $( \pm 0.5 \mathrm{~mm})$ were immersed in a dissolution bath containing $0.1 \mathrm{M} \mathrm{HCl}$ solution at $37^{\circ} \mathrm{C}$ with $50 \mathrm{rpm}$ paddle rotation speed. At regular time intervals, a piece was removed and cut in half, so a cross section became visible. Pictures were taken using a Thorlabs DCC1645C - High Resolution CMOS 
Camera (Thorlabs GmbH, Dachau/Munich, Germany). Hydrated zein matrix is opaque, whereas dry zein extrudates are yellow in colour. Using the colour change as a marker, the rate of hydration was estimated by measuring the change of the thickness of the hydrated layer with time. Image were analysed using Image-J software.

The hydration of the caplets was investigate using a conventional weighing method. Drug-loaded caplets were immersed in a dissolution bath containing dissolution media (either $\mathrm{HCl}$ or phosphate buffer) at $37^{\circ} \mathrm{C}$ with $50 \mathrm{rpm}$ paddle rotation speed. At regular time intervals, the sample was removed from the dissolution bath. Excess water was gently removed using a piece of paper towel before weighing the sample. After measuring the weight of the sample, it was immediately returned to the solution. The weight change of the sample was monitoring over a period of 54 hours in both $\mathrm{HCl}(\mathrm{pH}$ 1.2) and phosphate buffer (pH 6.8).

\section{Dissolution studies}

Caplets (diameter $5.9 \mathrm{~mm}$ ) were carefully cut into slices by the use of a razor blade. Slice thicknesses were $2.4 \pm 0.05 \mathrm{~mm}$ for most experiments, except for one experiment where slice thickness was varied. In this experiment a slice thicknesses of $1.1 \pm 0.05 \mathrm{~mm}$ and $4.5 \pm 0.05 \mathrm{~mm}$ were used additionally. Slice thicknesses and weights were measured using an electronic calliper and a microbalance respectively. Dissolution experiments were performed using the paddle method with $900 \mathrm{ml}$ dissolution media at $37.0 \pm 0.5^{\circ} \mathrm{C}$ and $50 \mathrm{rpm}$ rotation speed of the paddles.

Release kinetics were measured at regular time intervals. $10 \mathrm{ml}$ samples were taken and measured UV/vis spectrophotometer (S-22 Boeco, Boeckel and Co., Hamburg, Germany) at 270 $\mathrm{nm}$ corresponding to the $\lambda \max$ of paracetamol. 


\section{RESULTS AND DISCUSSION}

\section{Effect of hot melt extrusion on zein structure}

Zein is thermally stable for temperatures up to $120^{\circ} \mathrm{C}$, so the extrusion temperature of $80^{\circ} \mathrm{C}$ is not expected to effect the protein conformation significantly (9). However the combined stressing effect of increased temperature and shear force during HME may still alter the secondary structure of zein protein. To investigate this, ATR-FTIR spectroscopy was used to compare both untreated zein powder and extruded drug-free zein. Prior to the measurement, both samples were dried for 1 week in a desiccator at $0 \%$ relative humidity $(\mathrm{RH})$, to minimise the disruption of water peaks on the ATR-FTIT spectra of zein (water peak is broad and can overlap with the amide peak of zein protein). The deconvoluted and second derivative spectra of the amide I region, from $1580 \mathrm{~cm}^{-1}$ to $1700 \mathrm{~cm}^{-1}$, of both samples are displayed in Figure 1. From the second derivative results a clear absorption peak can be observed in both samples around $1650 \mathrm{~cm}^{-1}$, which is in line with earlier published data suggesting that the secondary structure of zein protein is dominated by $\alpha$-helices (17). In addition, peaks can be observed at 1615,1631 , 1681 and $1692 \mathrm{~cm}^{-1}$, which were also reported for zein (5). These four peaks are typically assigned to $\beta$-sheets of the zein protein (18). For the extruded samples, the $1650 \mathrm{~cm}^{-1}$ peak is less pronounced while the other peaks are increased. This suggests that during extrusion some of the $\alpha$-helical structure was lost, while more $\beta$-sheets were formed, which is consistent with other studies on processed zein (5). These changes in protein structure may be significant, since it has been reported that secondary structure can influence the drug release mechanism from zein based formulations (19). In this case intermolecular $\beta$-sheets could induce a network structure, potentially slowing down the water penetration and release of the incorporated drug. Although 
secondary structure of zein may have been slightly altered after HME, there is no evidence of degradation, indicating that the extrusion conditions used are appropriate for the purpose of this

study. This agrees well with the literature data in which zein protein crosslinking and chain cleavage are reported to occur at temperature above 120 and $180^{\circ} \mathrm{C}$, respectively (9). ATR-FTIR results of the HME zein matrix showed no significant changes after the follow-on injection moulding process (see Supplementary material). Therefore it is reasonable to conclude that both HME and IM lead to no degradation and little structure changes in zein.

\section{Microstructural and physicochemical characterisations of drug-loaded zein caplets and extrudates}

The physical state of the model drug in the zein matrix after extrusion and injection moulding may influence drug release behaviour and the caplet storage stability. When the drug is in an amorphous state or molecularly dispersed in zein, it can exhibit greater apparent solubility and subsequently increase the dissolution rate of the drug in the formulation (20). Therefore the processed samples were studied using SEM, PXRD and DSC to confirm the physical state of the model drug in the caplets.

Figure 2 shows the SEM images of the caplets surfaces and cross-sections. Between the samples with low drug loadings (4.5\% and 8.8\%) and high drug loadings (45.1\%), clear differences especially in the surface morphology can be observed. Relatively smooth surfaces without the presence of particle features can be seen for the caplets with low drug loading for both surface and cross-section. The samples with high drug loading (45.1\%) show rough outer surface, with visible particles in size of $\sim 20 \mu \mathrm{m}$. These particles may be crystalline paracetamol or phase 
separated domains containing drug and/or zein. To confirm the nature of these particles, further characterisation using DSC and PXRD were carried out and are discussed in the later sections. When comparing the caplet surface with the inner cross-section, for the sample with $45.1 \%$ paracetamol loading, more particles are seen in the inner core of the caplets.

Figure 3 shows the presence of clear crystalline drug diffraction peaks for the samples with high drug loadings (22.2\% and $45.1 \%$ ), whereas only single halo was observed for the samples with relatively low drug loadings (4.4\% and $8.8 \%$ ) indicating these samples being fully amorphous dispersions. This suggests the presence of crystalline paracetamol in the samples with high drug loadings (22.2\% and $45.1 \%$ ), which supports the interpretation that the particles seen in SEM are paracetamol crystals. The powder X-ray diffraction peaks from the caplets with $45.1 \%$ paracetamol loading are highly comparable to the diffraction pattern of pure crystalline paracetamol form I indicating the presence of high quantity of crystalline paracetamol form I. Although some peaks in this sample show anomalous intensity in comparison to the PXRD pattern of pure paracetamol form I, they do not resemble with other polymorphic forms of paracetamol (20). However for the $22.2 \%$ sample, in addition to the diffraction peaks of form I, new peaks were found at $22.2^{\circ}$ and $45.2^{\circ}$. These peaks are consistent with earlier reported PXRD diffraction pattern of crystalline paracetamol form III (21). This result indicates the presence of a mixture of crystalline forms I and III in the caplets with $22.2 \%$ drug loading. The presence of paracetamol form III was further confirmed by the DSC results.

Figure 4 shows the DSC results of the caplets with a range of different drug loadings. All loadings show a broad endothermic transition around $100^{\circ} \mathrm{C}$, which is likely due to moisture loss. Since zein absorbs moisture, it is likely to be taken up from the environment when 
preparing the sample prior to the DSC runs. For the samples with higher paracetamol loadings (22.2\% and $45.1 \%)$, melting peaks of crystalline paracetamol can be observed, which are absent for the formulations with lower drug loadings $(4.4 \%$ and $8.8 \%)$. This is in agreement with the PXRD results suggesting the amorphous nature of the formulations with low drug loadings and the presence of crystalline drug in the formulations with high drug loadings. For the $22.2 \%$ paracetamol loaded caplet, two endothermic peaks can be observed. One peak is at $163^{\circ} \mathrm{C}$, which is around the expected paracetamol form I melting temperature, and the other at $140^{\circ} \mathrm{C}$, which is at the reported melting temperature for paracetamol form III (22). This is in agreement with the PXRD results indicating the presence of form III in the $22.2 \%$ paracetamol loaded samples. Single broad melting with a peak temperature of $163^{\circ} \mathrm{C}$ can be seen in the DSC thermograph of the sample with $45.1 \%$ drug loading. Using the melting enthalpy of pure crystalline paracetamol $(181 \pm 0.7 \mathrm{~J} / \mathrm{g}$ for form I paracetamol), it is possible to estimate the amount of crystalline paracetamol in the sample. The caplet containing $45.1 \%$ paracetamol loading shows a melting enthalpy of $69.9 \mathrm{~J} / \mathrm{g}$. This suggests that approximately $85.7 \%(\mathrm{w} / \mathrm{w})$ of paracetamol present in this sample are in the crystalline form I. For the sample with $22 \%$ drug loading, the measured melting enthalpy of the $163^{\circ} \mathrm{C}$ peak is $1.7 \mathrm{~J} / \mathrm{g}$, indicating that only about $4.3 \%$ of the paracetamol is in crystalline form I. The estimated melting enthalpy of the form III paracetamol is $8.5 \mathrm{~J} / \mathrm{g}$ suggesting about $23.2 \%$ of the paracetamol is present in form III as calculated from the earlier reported form III melting enthalpy being $165 \mathrm{~J} / \mathrm{g}(23)$.

We hypothesise that there may be limited dissolution of the paracetamol in the water (10-12\% w/w) used during the pre-mixing prior to extrusion. However as the water was mixed with zein prior to the adding of paracetamol, majority of water is expected to contribute to the zein hydration instead of drug dissolution. Nevertheless it is reasonably to predict that small quantity 
of paracetamol may be dissolved during the pre-mixing stage and molecularly dispersed in the partially hydrated zein. Further loss of paracetamol crystallinity could be due to dissolution into the plasticised (by water) zein matrix during extrusion at the processing temperature above the $\mathrm{T}_{\mathrm{g}}$ of the zein/water matrix (24). Under this circumstance, the drug-polymer solubility may play an important role in inhibiting the recrystallization of molecularly dispersed drug in the polymer matrix. The fact that the formulations with low drug loadings (4.4 and $8.8 \%$ ) showed no sign of the presence of crystalline drug indicates that these two drug loadings are likely to be at or below the solubility of paracetamol in zein processed by HME-IM. The model drug is likely to be present as molecular dispersion or phase separate amorphous drug-rich domains in the processed formulations. However, the presence of crystalline paracetamol in the $22.2 \%$ drug loaded caplets suggests that this drug loading is likely to be above the drug-polymer solubility, which leads to the rapid drug recrystallization after HME-IM. The dissolved paracetamol during HME-IM may reach the saturation of paracetamol in zein and rapidly recrystallize on cooling. It is highly likely that the recrystallization into metastable form III occurred prior to the solid state transformation of form III into the stable form I. Initial recrystallization into metastable polymorphic form is common and has been reported for paracetamol (25). Therefore both polymorphic form I and III were detected in the caplets with $22.2 \%$ drug loading. In the $45.1 \%$ sample, a significant amount of paracetamol crystalline form I was detected in the processed formulation. We suspect that some of the form I crystals remained un-dissolved in the matrix during the HME-IM at $80^{\circ} \mathrm{C}$ and these form I crystals can act as nucleus and promote the polymorphic conversion of form III into form I. Therefore only form I is observed in the DSC and PXRD results of the $45.1 \%$ samples. The confirmation of the presence of crystalline paracetamol by $45.1 \%$ caplets by both DSC and 
PXRD results suggests that the partials observed in the SEM images of the sample are highly likely to be crystalline paracetamol particles.

\section{Hydration kinetics of drug-loaded zein extrudates}

The zein-based extrudates and caplets developed in this study are single-unit matrix systems, which do not exhibit disintegration behaviour prior to dissolution. The drug release was accompanied by the swelling (expansion in volume) and hydration (water uptake) of the formulations. They play an important role in modulating the drug release. Therefore the kinetics of the swelling (measuring the volume change v.s. time) and hydration (measuring the weight change v.s. time) behaviour of the formulations was studied. During hydration and swelling, zein loses its distinct yellow colour and turns pale. This property was used to follow the swelling kinetics of drug-loaded zein extrudates during dissolution. At various time intervals, piece of the extrudate were removed and cut in half, so a fresh cross section could be revealed. From these cross sections the wet pale outer layer, whose thickness increased with time, became visible, as displayed in Figure 5a which is the result of the extrudates with $8.8 \%$ drug loading. Using image analysis, normalised values for the inner radius and the outer radius were obtained (Figure 5b). Similar images with a slower rate of swelling were obtained for the extrudates immersed in phosphate buffer (data not shown).

The extrudate swells up to approximately 1.5 times its original size after 48 hours immersion in $\mathrm{HCl}$. It can also be observed that the colour gradient between the pale outside and the darker inner core remains sharp. This sharp boundary between the swelled outer layer and relatively dry inner glassy polymer core is typical for Case II diffusion, in which this boundary moves at a constant velocity $(26,27)$. This is reasonably consistent with the data presented in Figure 5 b, in 
which the rates of changes of the thickness of the outer layer and inter core are linear. Different models have been proposed in literature to explain this phenomenon $(26,27)$. For example, Thomas and Windle (TW) model considers the swelling of the outer plasticized polymer (by water in this case) shell is driven by osmotic pressure and the movement of the sharp diffusion front is controlled by the coupling of osmotic-pressure-driven swelling and nearly Fickian diffusion in the glassy polymer core (27). Recently the sharp diffusion front was also explained by a 'change of state' theory, in which it assumes that when the freely diffused small solvent molecules reach the glassy polymer core (diffusion front) they are partially immobilised (via adsorption or binding) at the surface of the holes and microvoids of glassy polymer. The significant reduction on the mobility of the small molecules at the boundary interface results the sharp diffusion front (28). In either case the observable swelling and water uptake phenomena are the same.

The water uptake by the drug-loaded zein-based formulations was further quantified using the conventional weighing method. It can be seen in Figure 6 that caplets in $\mathrm{HCl}$ solution took up almost twice the amount of water compared to the ones immersed in phosphate buffer. The deamidation of the glutamine and asparagine could occur in the acidic media and cause the zein network to loosen up and allow more water to be incorporated into the swollen zein matrix (29). The higher water uptake for the caplets in the $\mathrm{HCl}$ media may be explained by this mechanism. This could cause the diffusivity of the paracematol in the zein network to increase, thus resulting in faster dissolution rates in comparison to the results obtained using phosphate buffer media. This is confirmed by the in vitro drug release data shown below . 


\section{Evaluation of tuneability of in vitro drug release}

The tuneability of drug release from zein-based caplets was studied in vitro by altering the drug loading and dimensions of the caplets. In Figure 7, the drug release profiles of the slices of zein caplets are displayed for four different drug loadings in two different dissolution media. The complete drug release was achieved over 30 hours in both media without initial burst release (see Supplementary material), indicating that the zein matrix is a suitable candidate excipient for the preparation of sustained drug delivery systems. It can be observed in Figure 7 that the drug release is slower in phosphate buffer compared to $0.1 \mathrm{M} \mathrm{HCl}$. This could be attributed to the slower hydration rate and lower hydration values found in the weight based hydration experiments.

Slightly faster release rates can be observed for the samples with lower paracetamol loadings (4.4\% and $8.8 \%$ ) compared to the higher paracetamol loadings $(22.2 \%$ and $45.1 \%)$. This may be partially attributed to the fact that paracetamol is molecularly dispersed in this formulations as indicated by the previous solid-state characterisation results. However, in the samples with higher drug loadings containing crystalline paracetamol, the dissolution of paracetamol crystals can form pores in the zein matrix, which could increase the sample porosity and subsequently the water penetration rates. This effect will be partially compromised again by the swelling of the zein which will close the pore. Although paracetamol crystals are present in the samples with high drug loadings, the thermodynamic driving force for dissolution is higher due to the high drug concentration in the matrix in comparison to the systems with lower drug loadings. There is therefore a balance of forces acting. Overall the release rates of the samples show weak 
dependence on paracetamol loading, indicating that the zein matrix can retain its slow release functionality for paracetamol concentrations up to $45.1 \%$.

Model-fitting of the drug release data was performed in an attempt to reveal the dominating mechanism of the drug release from the zein-based caplets. In Figure 7, the inserted log-log plots of the drug release data show a linear correlation, strongly suggesting a power law relationship applies. One of the well-known power law equations to explain the mechanism of drug release is the Peppas and Sahlin equation $(29,30)$.

$$
\frac{M_{t}}{M_{\infty}}=k t^{n}
$$

where $M_{t}$ is the mass of drug released at time $t$ and $M_{\infty}$ is the mass of drug release as time approaches infinity. Parameter $t$ is time in minutes, $k$ is a constant incorporating characteristics of the macromolecular network and the drug, and $n$ is a diffusional exponent indicative for the transport mechanism. The value of $n$ is dependent both on the geometry of the sample and the nature of the diffusion process. For the cylindrical geometry used in this study with a slice thickness-to-diameter ratio of 2.5 , there are two limits to $n$ : when the value $n$ is 0.45 , the drug release is dominated by molecular diffusion which is driven by a gradient in chemical potential (Fickian diffusion) (30). For this geometry when the value of $n$ approaches 0.9 , diffusion can be described by Case II diffusion, which is commonly associated with the glassy-to-gel state transition and relaxation of hydrophilic polymers which swell in water or biological fluids (30). In most cases the value of $n$ is in between 0.45 and 0.9 in which a combination of Fickian diffusion and Case II transport applies. This type is often referred to as anomalous release. The power-law approximation was initially thought to be only valid in the short time limit (33). 
However, later it was shown (33) the power-law approximation is valid up to 0.6 total fractional drug release. This means that the diffusional exponent $n$ can only be correctly defined, when data fits are performed below 0.6 total fractional drug release. Model fitting results for the fractional drug release data up to 0.6 is shown in Table, where values of $n$ and $k$ are given. It can be observed that values of $n$ are between 0.52 and 0.62 indicating anomalous release applies with a strong tendency to Fickian controlled release mechanism. This is further examined by comparing the rate of drug release with the rate of hydration of the drug-loaded caplets.

It can be observed in Figure 5c that hydration occurred at a significantly faster rate than the drug release of the same formulation. This suggests that the diffusion of paracetamol (carried out by the dissolution media) through the hydrated extrudate is slower than the hydration of the zein matrix and is the main rate-limiting factor for release. Therefore it is reasonable to predict that release would be driven purely by Fickian diffusion with a resulting diffusional exponent $n$ of 0.45 . However the swelling caused by water absorption results in a continuous change in the boundary conditions for diffusion which accounts for the deviation from the ideal exponent. In addition as pointed out earlier for the samples with crystalline material present the dissolution of the crystals result in a higher porosity and surface area for drug release. This explains the slightly higher $(n>0.45)$ exponent values observed for the systems studied.

Finally to quantify the influence of caplet dimensions on the drug release profile, dissolution tests on caplet slices (diameter $6 \mathrm{~mm}$ ) with three different lengths were performed. As seen in Figure 8, the release rate decreases with increasing the caplet length. All samples show good linear correlation of release v.s. square root of time. This indicates that the drug release is 
predominately governed by Fickian diffusion (34). Table 2 shows the power-law fitting results and half-life $\left(t_{0.5}\right)$ of the drug release for the samples with three different lengths. Fittings were only performed up to total fractional drug release of 0.6. From the $n$-values shown in Table 2, a correlation can be found between slice length $(l)$ and constant $(k)$, which is confirmed when comparing $t_{0.5}$ with slice length. The diffusional exponent $n$ stays broadly the same with the change in dimensions indicating the release mechanism remains the same regardless the change of the dimensions of the drug delivery device.

In an earlier study by Ritger and Peppas (33) an exact expression was derived, describing release from disk-like geometries up to 0.9 fractional drug release:

$$
\frac{M_{t}}{M_{\infty}}=4\left[\frac{D t}{\pi a^{2}}\right]^{\frac{1}{2}}-\pi\left[\frac{D t}{\pi a^{2}}\right]-\frac{\pi}{3}\left[\frac{D t}{\pi a^{2}}\right]^{\frac{3}{2}}+4\left[\frac{D t}{\pi a^{2}}\right]^{\frac{1}{2}}-\frac{2 a}{l}\left[8\left(\frac{D t}{\pi a^{2}}\right)-2 \pi\left(\frac{D t}{\pi a^{2}}\right)^{\frac{3}{2}}-\frac{2 \pi}{3}\left(\frac{D t}{\pi a^{2}}\right)^{2}\right]
$$

In this model the one-dimensional release equations of both slabs and long cylinders are summed up and additionally a coupling term was incorporated. $M_{t}$ is the mass of drug released at time $t$ and $M_{\infty}$ is the mass of drug release as time approaches infinity, $D$ is the diffusion coefficient. The symbols $a$ and $l$ are the disk radius and thickness respectively.

By fitting the data to this model, the mutual effective diffusion coefficient $D$ of paracetamol in hydrated zein could be approximated. In Table it can be observed that $D$ is around $5^{*} 10^{-12} \mathrm{~m}^{2} / \mathrm{s}$. Both the low variation in $D$ for the different caplet lengths and the high correlation coefficient $\left(R^{2}\right)$ values indicate that this model is highly suitable to explain the dependency of release kinetics on the device dimensions of this study. The value found for $D$ is approximately 100 times higher than the diffusion coefficient of paracetamol in water $\left(\sim 4 * 10^{-10} \mathrm{~m}^{2} / \mathrm{s}\right)$ estimated 
using the Stokes-Einstein relationship. This is a clear indication of reduced diffusion rate of dissolved paracetamol in hydrated zein matrix. The estimate of the effective diffusion coefficient allows the estimation of the release rates achievable by changing the device dimensions. Further manipulation of drug release may be made by the use of different plasticisers. However it should be noted that different drugs may have different effect on the release rates because molecular dimensions will result in changes in diffusion in the zein matrix. It is also noted in Table 2 that the half-life increased by 6 fold when the length of the extrudates increased from 1.1 to $4.5 \mathrm{~mm}$. In comparison to this level of change, the changes in half-life observed in the samples with different drug loading is significantly less, being less than 0.3 fold when increased the drug loading from 4.4 to $45.1 \%$. These results indicate that stronger dimension effect on the drug release kinetics than the drug loading.

\section{CONCLUSION}

In this study paracetamol was successfully encapsulated into Fickian diffusion controlled drug delivery devices of zein by the use direct HME-IM. For lower drug loadings (4.4\% and $8.8 \%)$ paracetamol is present as molecular dispersion in the zein matrix, whereas crystalline paracetamol are present in the formulations with higher drug loadings (22.2\% and $45.1 \%)$. The drug release results confirmed that the zein caplets are suitable for controlled release, where release was found to be weakly dependent on drug loading but strongly dependent upon formulation dimensions. Both the power law fits to the release data as the real time hydration data indicated Fickian diffusion release to be the dominating mechanism. The strong dependence of drug release on formulation dimension can be used to manipulate the release rate by simply altering the dimensions of the device. The results of this study demonstrated the potential of 
using zein, a natural polymer from a highly sustainable source, as the sole excipient to create a Fickian diffusion controlled device suitable for tuneable controlled release by simple solvent-free HME-IM method.

\section{ACKNOWLEDGEMENTS}

This work is part of the Industrial Partnership Programme (IPP) Bio(-Related)Materials of the Stichting voor Fundamenteel Onderzoek der Materie (FOM), which is financially supported by the Nederlandse Organisatie voor Wetenschappelijk Onderzoek (NWO). The IPP BRM is cofinanced by the Top Institute Food and Nutrition and the Dutch Polymer Institute. 


\section{REFERENCES}

1. K.E. Uhrich, S.M. Cannizzaro, R.S. Langer, and K.M. Shakesheff. Polymeric Systems for Controlled Drug Release. Chemical Reviews. 99:3181-3198 (1999).

2. S. Grund, M. Bauer, and D. Fischer. Polymers in drug delivery-state of the art and future trends. Advanced Engineering Materials. 13:B61-B87 (2011).

3. X. Liu, Q. Sun, H. Wang, L. Zhang, and J.Y. Wang. Microspheres of corn protein, zein, for an ivermectin drug delivery system. Biomaterials. 26:109-115 (2005).

4. H.-J. Wang, Z.-X. Lin, X.-M. Liu, S.-Y. Sheng, and J.-Y. Wang. Heparin-loaded zein microsphere film and hemocompatibility. Journal of Controlled Release. 105:120-131 (2005).

5. D.M.R. Georget, S.A. Barker, and P.S. Belton. A study on maize proteins as a potential new tablet excipient. European Journal of Pharmaceutics and Biopharmaceutics. 69:718726 (2008).

6. J.W. Lawton. Zein: A history of processing and use. Cereal Chemistry. 79:1-18 (2002).

7. Q. Wang, L. Yin, and G. Padua. Effect of Hydrophilic and Lipophilic Compounds on Zein Microstructures. 3:174-181 (2008).

8. P.S. Belton, I. Delgadillo, N.G. Halford, and P.R. Shewry. Kafirin structure and functionality. Journal of Cereal Science. 44:272-286 (2006).

9. G.W. Selling. The effect of extrusion processing on Zein. Polymer Degradation and Stability. 95:2241-2249 (2010).

10. R. Paliwal, S. Palakurthi. Zein in controlled drug delivery and tissue engineering. J Control Release. 189:108-22 (2014)

11. K. Takagi, R. Teshima, H. Okunuki, and J.I. Sawada. Comparative study of in vitro digestibility of food proteins and effect of preheating on the digestion. Biological and Pharmaceutical Bulletin. 26:969-973 (2003).

12. M. Zhang, C.A. Reitmeier, E.G. Hammond, and D.J. Myers. Production of textile fibers from zein and a soy protein-zein blend. Cereal Chemistry. 74:594-598 (1997).

13. G.W. Selling, K.K. Woods, A. Biswas, and J.L. Willett. Reactive extrusion of zein with glyoxal. J Appl Polym Sci. 113:1828-1835 (2009).

14. L. Eith, R.F.T. Stepto, I. Tomka, and F. Wittwer. The Injection-Moulded Capsule. Drug Development and Industrial Pharmacy. 12:2113-2126 (1986).

15. J.S. Deng, M. Meisters, L. Li, J. Setesak, L. Claycomb, Y. Tian, D. Stephens, and M. Widman. The development of an injection-molding process for a polyanhydride implant containing gentamicin sulfate. PDA Journal of Pharmaceutical Science and Technology. 56:65-77 (2002).

16. R.K. Gilpinand W. Zhou. Studies of the Thermal Degradation of Acetaminophen Using a Conventional HPLC Approach and Electrospray Ionization-Mass Spectrometry. 42:15-20 (2004).

17. L.A. Forato, T.D.C. Bicudo, and L.A. Colnago. Conformation of $\alpha$ Zeins in Solid State by Fourier Transform IR. Biopolymers - Biospectroscopy Section. 72:421-426 (2003).

18. D.M.R. Georgetand P.S. Belton. Effects of Temperature and Water Content on the Secondary Structure of Wheat Gluten Studied by FTIR Spectroscopy. Biomacromolecules. 7:469-475 (2006). 
19. J.Y. Fang, J.P. Chen, Y.L. Leu, and H.Y. Wang. Characterization and evaluation of silk protein hydrogels for drug delivery. Chemical and Pharmaceutical Bulletin. 54:156-162 (2006).

20. C. Ahlneckand G. Zografi. The molecular basis of moisture effects on the physical and chemical stability of drugs in the solid state. International Journal of Pharmaceutics. 62:87-95 (1990).

21. M.L. Peterson, S.L. Morissette, C. McNulty, A. Goldsweig, P. Shaw, M. LeQuesne, J. Monagle, N. Encina, J. Marchionna, A. Johnson, J. Gonzalez-Zugasti, A.V. Lemmo, S.J. Ellis, M.J. Cima, and Ö. Almarsson. Iterative High-Throughput Polymorphism Studies on Acetaminophen and an Experimentally Derived Structure for Form III. Journal of the American Chemical Society. 124:10958-10959 (2002).

22. A. Rossi, A. Savioli, M. Bini, D. Capsoni, V. Massarotti, R. Bettini, A. Gazzaniga, M.E. Sangalli, and F. Giordano. Solid-state characterization of paracetamol metastable polymorphs formed in binary mixtures with hydroxypropylmethylcellulose. Thermochimica Acta. 406:55-67 (2003).

23. T.R. Gopalakrishnan. Polymorphism of acetaminophen under nanoconfinement, Naturwissenschaftliche Fakultät II, Martin-Luther-Universität Halle-Wittenberg, Halle, 2010.

24. T. Gillgren, S.A. Barker, P.S. Belton, D.M.R. Georget, and M. Stading. Plasticization of zein: A thermomechanical, FTIR, and dielectric study. Biomacromolecules. 10:11351139 (2009).

25. S. Qi, P. Avalle, R. Saklatvala, and D.Q.M. Craig. An investigation into the effects of thermal history on the crystallisation behaviour of amorphous paracetamol. European Journal of Pharmaceutics and Biopharmaceutics. 69:364-371 (2008).

26. A. Peterlin. Diffusion in a network with discontinuous swelling. J Polym Sci B Polym Lett. 3:1083-1087 (1965).

27. N.L. Thomasand A.H. Windle. A theory of case II diffusion. Polymer. 23:529-542 (1982).

28. M.O. Gallyamov. Sharp diffusion front in diffusion problem with change of state. Eur Phys J E Soft Matter. 36(8):92 (2013).

29. Y.H. Yong, S. Yamaguchi, Y.S. Gu, T. Mori, and Y. Matsumura. Effects of Enzymatic Deamidation by Protein-Glutaminase on Structure and Functional Properties of $\alpha$-Zein. Journal of Agricultural and Food Chemistry. 52:7094-7100 (2004).

30. N.A. Peppas and J.J. Sahlin. A simple equation for the description of solute release. III. Coupling of diffusion and relaxation. International Journal of Pharmaceutics. 57:169-172 (1989).

31. H.L. Frisch. Sorption and transport in glassy polymers-a review. Polym Eng Sci. 20:2-13 (1980).

32. T. Alfrey, E.F. Gurnee, and W.G. Lloyd. Diffusion in glassy polymers. J polym sci, C Polym symp. 12:249-261 (1966).

33. P.L. Ritgerand N.A. Peppas. A simple equation for description of solute release I. Fickian and non-fickian release from non-swellable devices in the form of slabs, spheres, cylinders or discs. Journal of Controlled Release. 5:23-36 (1987).

34. R.B. Bird, W.E. Stewart, and E.N. Lighfoot. Transport phenomena, John Wiley, Estados Unidos, 1976. 
Table 1. Fitting parameters of the drug release data of caplets with different drug loadings and in different media using power-law. Half-life values were determined using the fitting parameters.

\begin{tabular}{cccccc}
\hline Dissolution medium & Drug loading $\mathbf{( \% )}$ & $\mathbf{k} \cdot \mathbf{1 0}^{\mathbf{2}}\left(\mathbf{m i n}^{-\mathbf{n}}\right)$ & $\mathbf{n}$ & $\mathbf{R}^{\mathbf{2}}$ & $\mathbf{t}_{\mathbf{0 . 5}}(\mathbf{m i n})$ \\
\hline $\mathrm{HCl}$ solution & 4.4 & $2.3 \pm 0.5$ & $0.52 \pm 0.03$ & 0.985 & 373 \\
$\mathrm{pH}=1$ & 8.8 & $1.9 \pm 0.6$ & $0.55 \pm 0.02$ & 0.966 & 382 \\
& 22.2 & $1.6 \pm 0.1$ & $0.59 \pm 0.01$ & 0.999 & 342 \\
& 45.1 & $1.2 \pm 0.1$ & $0.62 \pm 0.02$ & 0.998 & 410 \\
\hline Phosphate buffer & 4.4 & $1.4 \pm 0.1$ & $0.54 \pm 0.01$ & 0.999 & 751 \\
pH=6.8 & 8.8 & $1.0 \pm 0.1$ & $0.60 \pm 0.02$ & 0.994 & 679 \\
& 22.2 & $1.3 \pm 0.1$ & $0.58 \pm 0.01$ & 0.999 & 541 \\
& 45.1 & $1.3 \pm 0.1$ & $0.59 \pm 0.01$ & 0.998 & 486 \\
\hline
\end{tabular}

Table2. Fitting parameters of the drug release data of $8.8 \%$ drug-loaded caplets with different slice lengths (shown in Figure 8) using Eq. 1. Half-life values were determined using the fit parameters. Effective diffusion coefficients $D$ were determined by fitting these release data to Eq. 2.

\begin{tabular}{c|rccccc}
\hline $\begin{array}{c}\text { Slice length } \\
(\mathbf{m m})\end{array}$ & $\mathbf{k} \cdot \mathbf{1 0}^{\mathbf{2}}\left(\mathbf{m i n}^{-\mathbf{n}}\right)$ & $\mathbf{n}$ & $\mathbf{R}^{\mathbf{2}}$ & $\mathbf{t}_{\mathbf{0 . 5}}(\mathbf{m i n})$ & $\mathbf{D} \cdot \mathbf{1 0} \mathbf{1 0}^{\mathbf{1 2}}\left(\mathbf{m}^{\mathbf{2}} / \mathbf{s}\right)$ & $\mathbf{R}^{\mathbf{2}}$ \\
\hline 1.1 & $2.77 \pm 0.4$ & $0.57 \pm 0.02$ & 0.998 & 160 & $4.8 \pm 0.4$ & 0.985 \\
2.3 & $1.86 \pm 0.2$ & $0.55 \pm 0.02$ & 0.966 & 407 & $5.0 \pm 0.2$ & 0.990 \\
4.5 & $1.69 \pm 0.2$ & $0.51 \pm 0.01$ & 0.998 & 766 & $5.3 \pm 0.2$ & 0.994 \\
\hline
\end{tabular}


Figure 1: ATR-FTIR spectra for dried zein powder (DZP) and dried ground zein extrudates (DGZE). Solid lines are the baseline corrected spectra of DZP (dark) and DGZE (grey). Dashed lines are the secondary derivatives of DZP (dark) and DGZE (grey). Detailed assignments of different forms of secondary structures of zein protein are shown in the secondary derivative spectra.

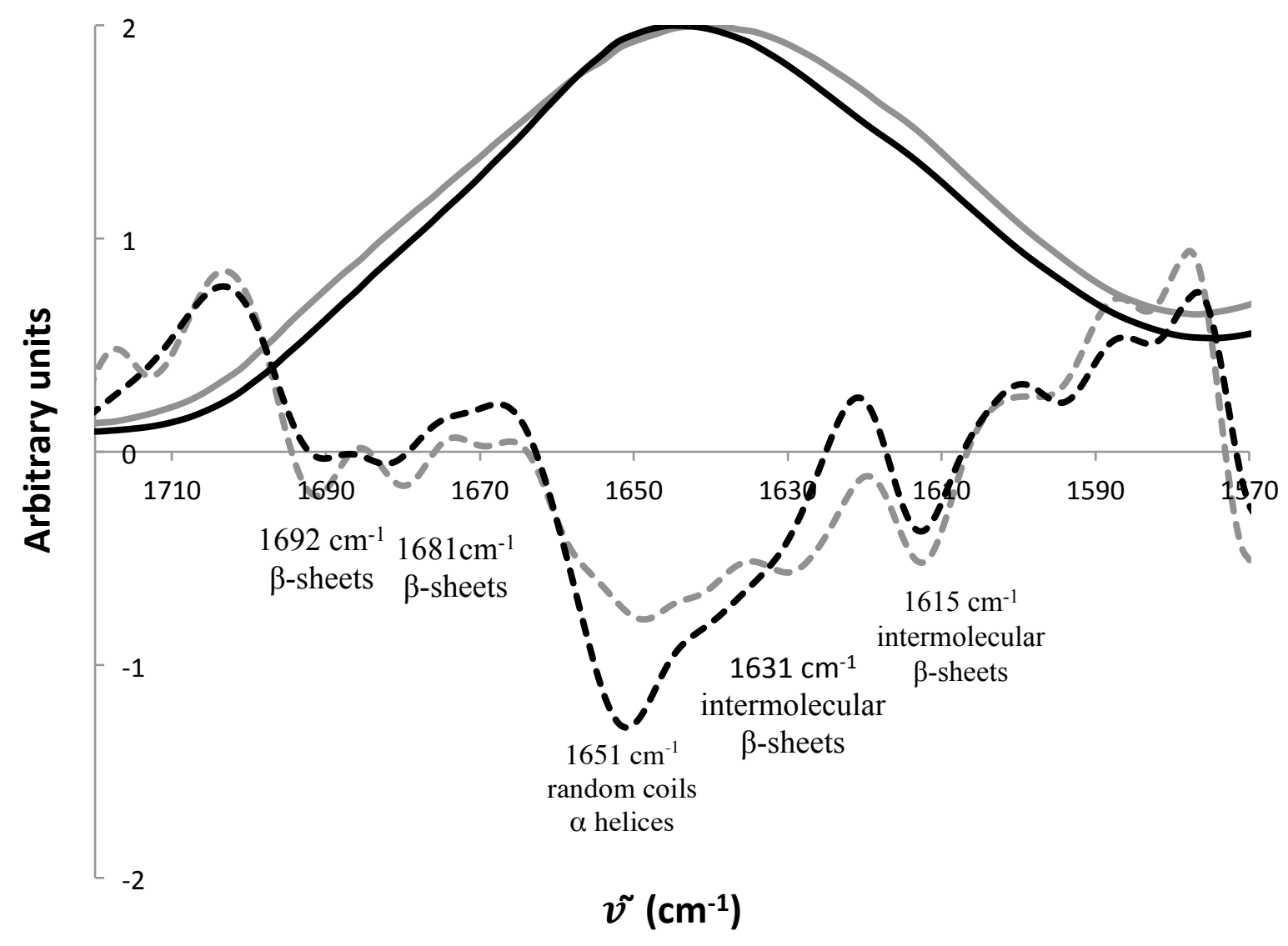


Figure 2: SEM images of the cross-sections of the inner core and outer surfaces of the caplets with two different loadings.

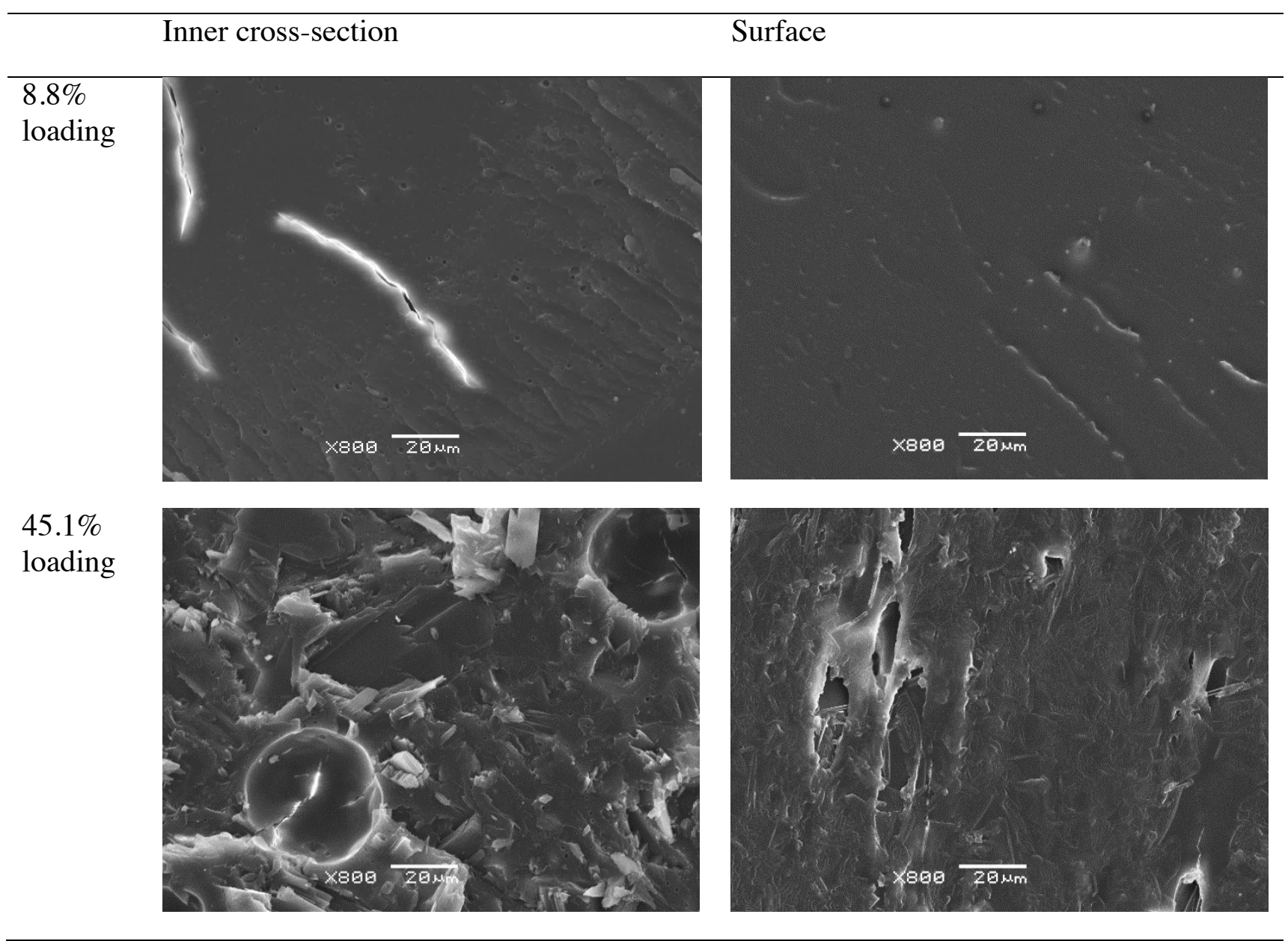


Figure 3: PXRD spectra of dried zein/paracetamol caplet slices with a) $45.1 \%$, b) $22.2 \%$, c) $8.8 \%$ and d) $4.4 \%$ drug loadings compared with p) crystalline paracetamol form I powder.

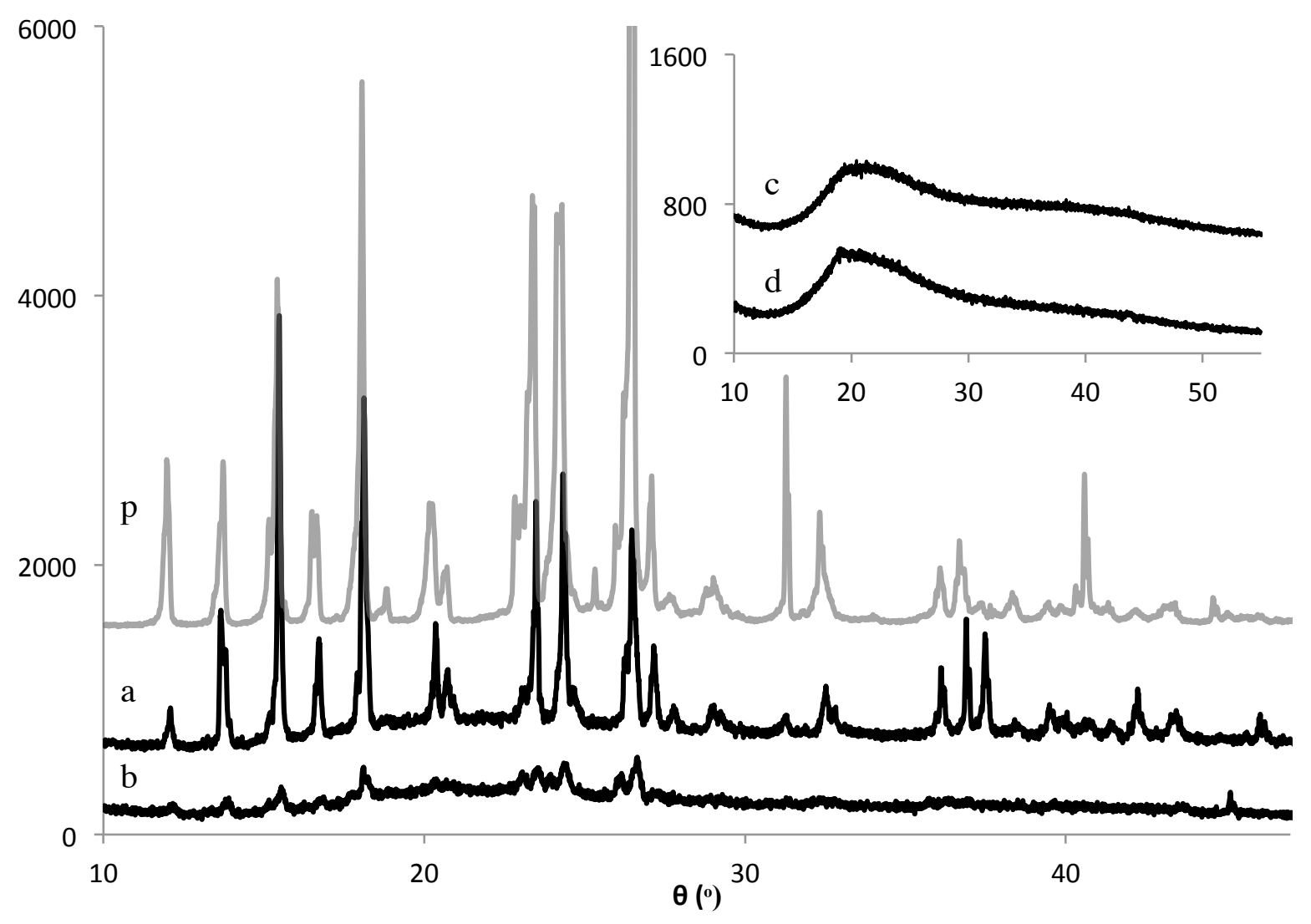


Figure 4: DSC thermograms of the dried zein/paracetamol caplets. Caplets with 4.4\% paracetamol loadings (grey dashed line); $8.8 \%$ (black dashed line); 22.2\% (grey solid line) and $45.1 \%$ (black solid line).

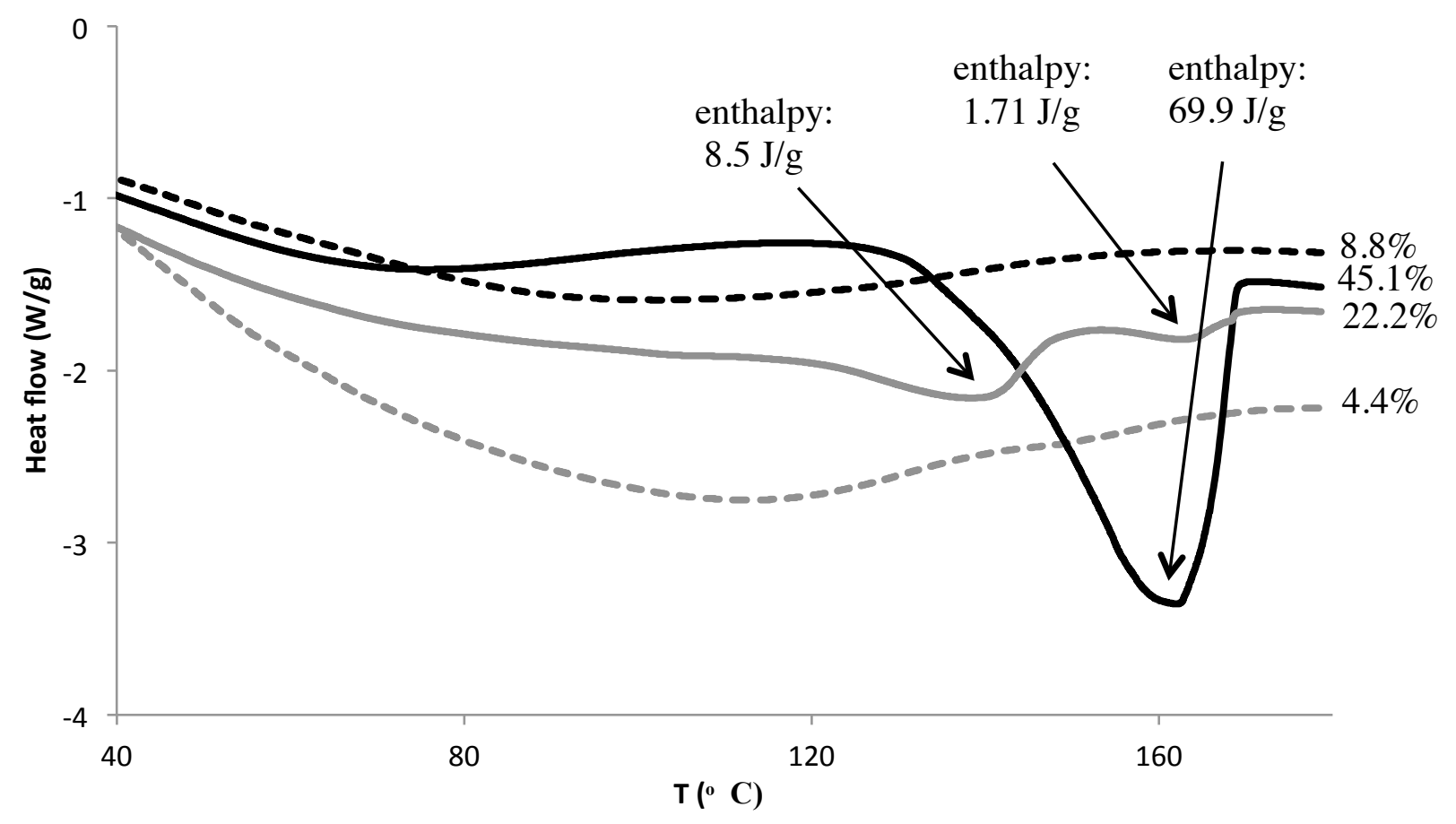


Figure 5: Swelling results of the cylindrical extrudates with $8.8 \%$ drug loading immersed in 0.1 $\mathrm{M} \mathrm{HCl}$. a) The cross-section images of the extrudates after different hydration times; b) image analysis results: initial radius/measured $\left(\mathrm{r} / \mathrm{r}_{0}\right)$ versus time $(\mathrm{t})$ (outer radius-closed squares and inner radius-open diamonds); (c) drug release (closed squares) and hydration (open diamonds) fractions are plotted against time for cylindrical extrudate with $8.8 \%$ drug loading in $0.1 \mathrm{M} \mathrm{HCl}$.

(a)

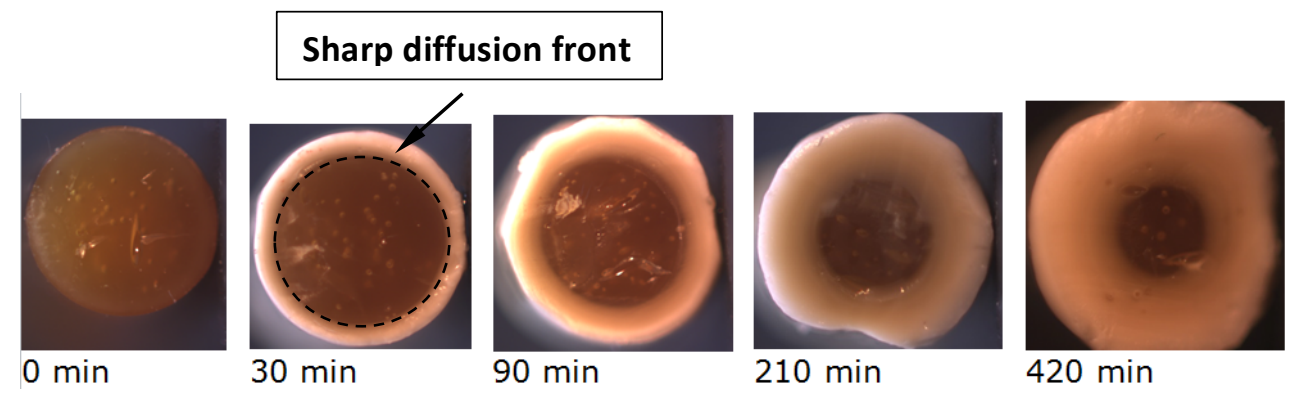

(b)

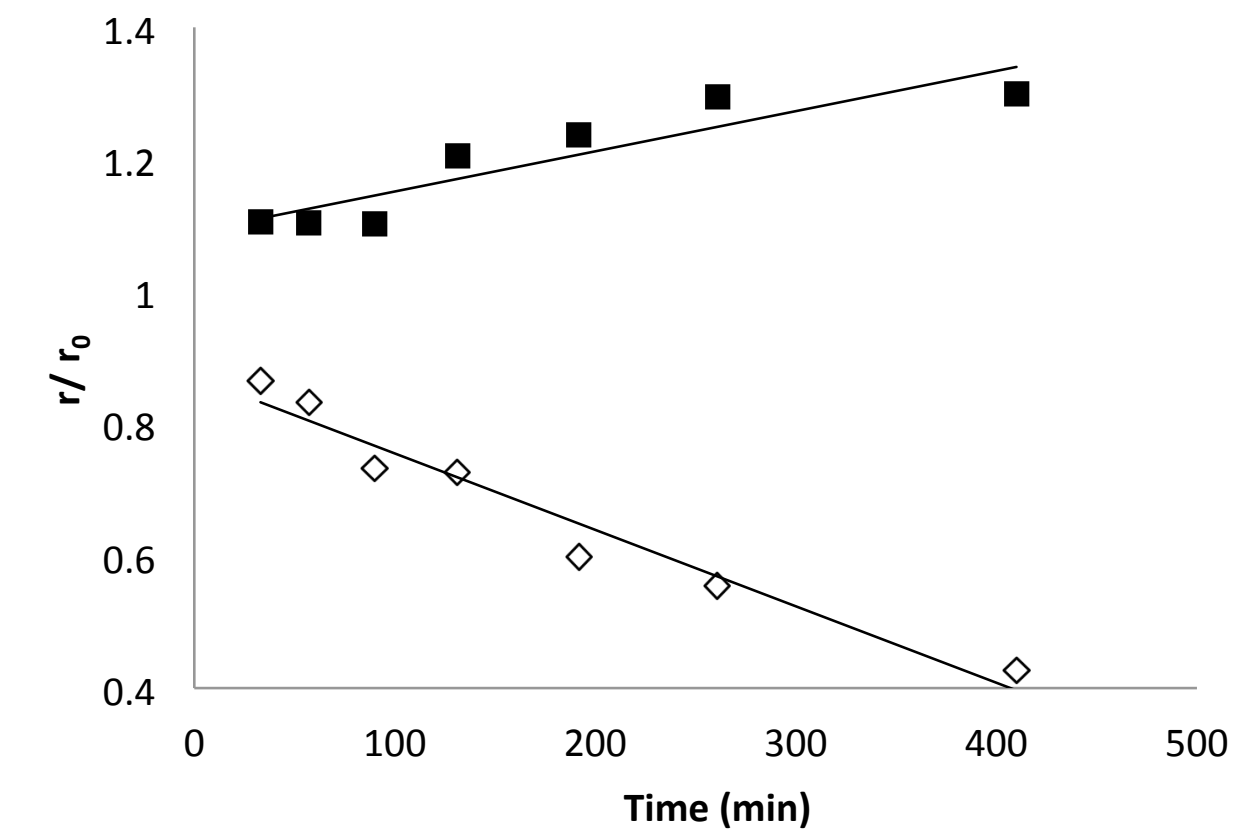




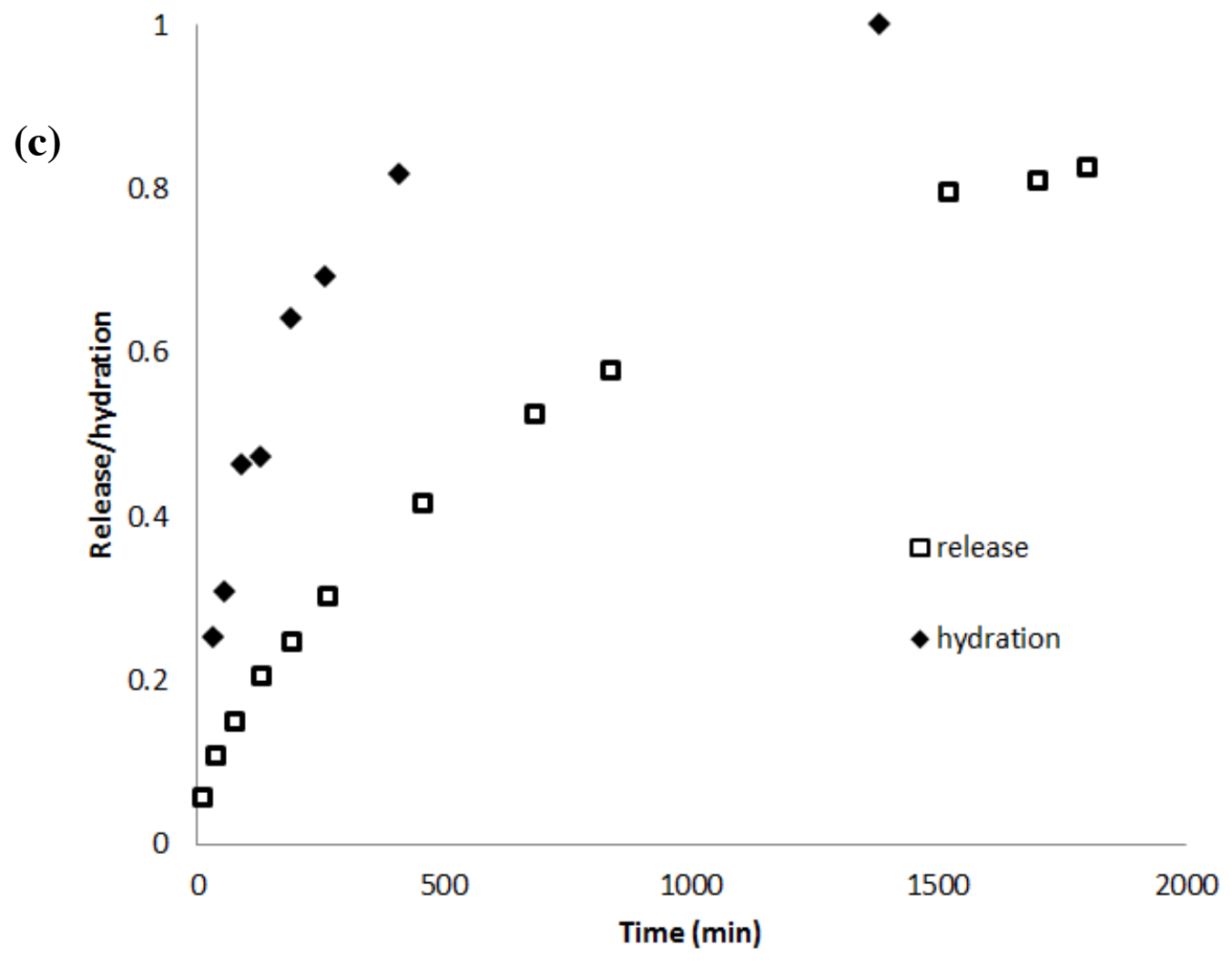


Figure 6: Monitoring of the hydration behaviour of caplets with $8.8 \%$ drug loading in $\mathrm{HCl}(\mathrm{pH}$ 1.2) and phosphate buffer (pH 6.8) using the weight based method.

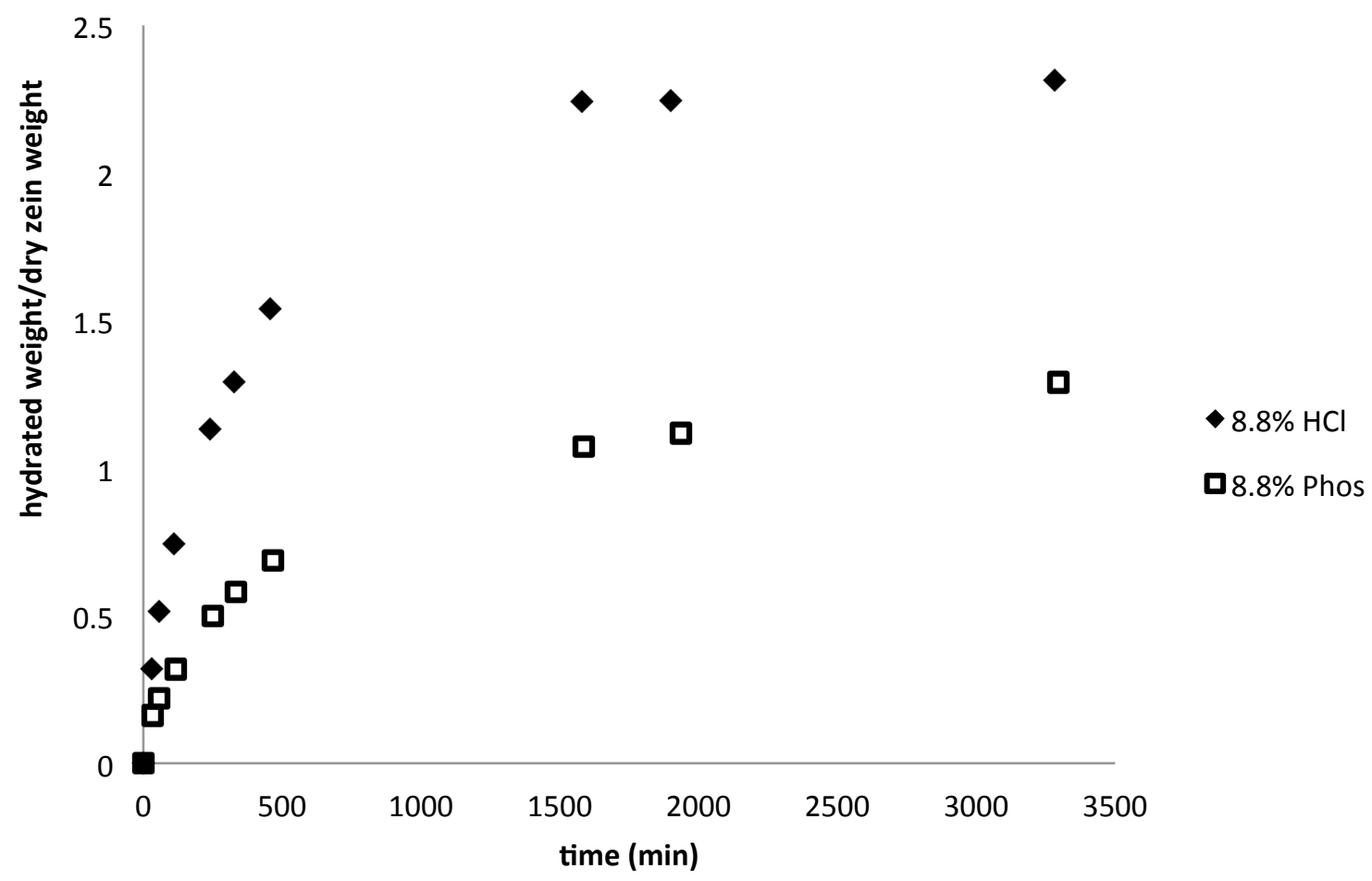


Figure 7: Release profiles for the caplets with different drug loadings (\%) in (a) $0.1 \mathrm{M} \mathrm{HCl}$ and (b) phosphate buffer ( $\mathrm{pH}$ 6.8). Insert shows selected profiles fitted with a power-law function (equation [2]) and the $\mathrm{Y}$ - and $\mathrm{X}$-axis are on log scale.

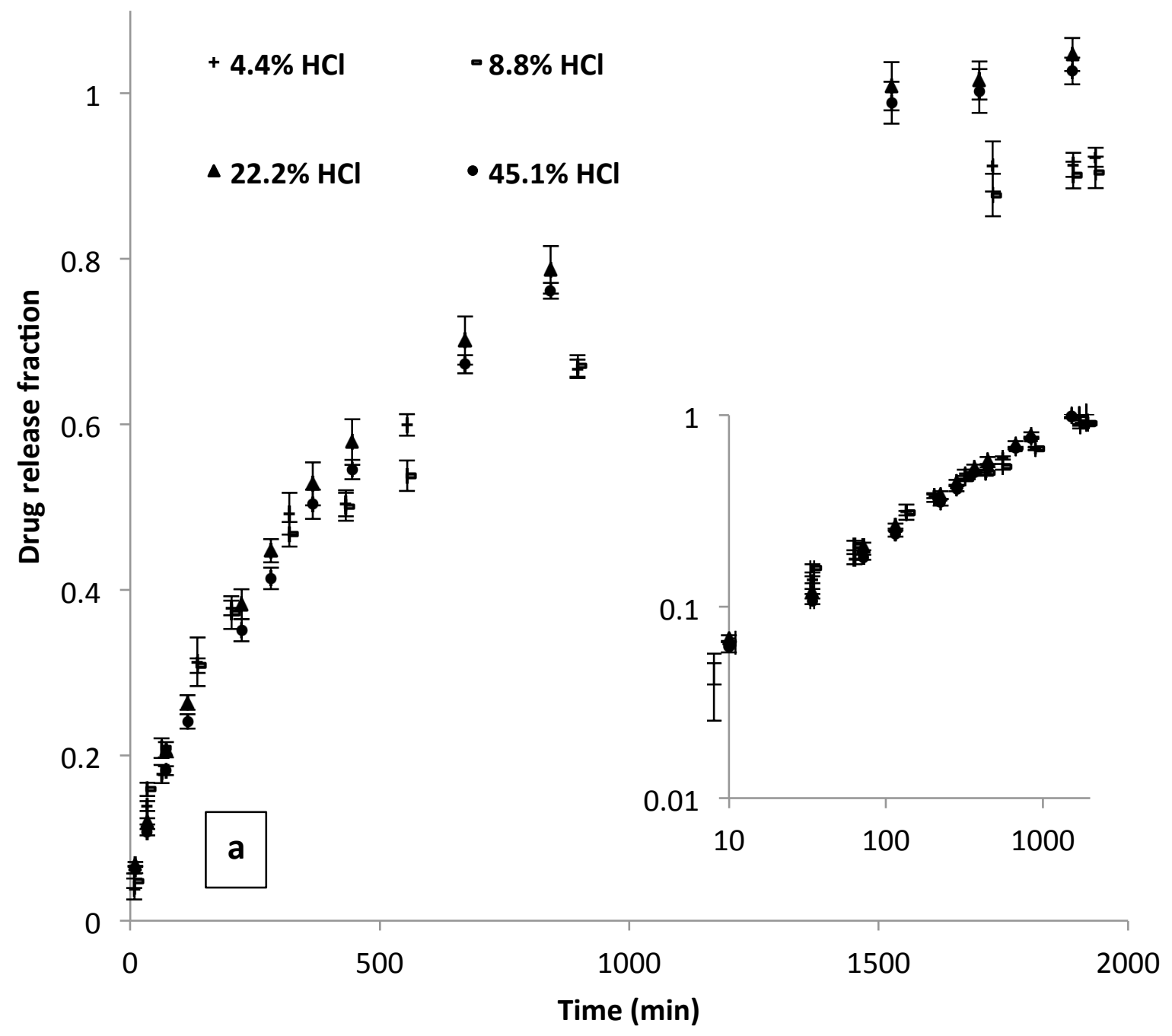




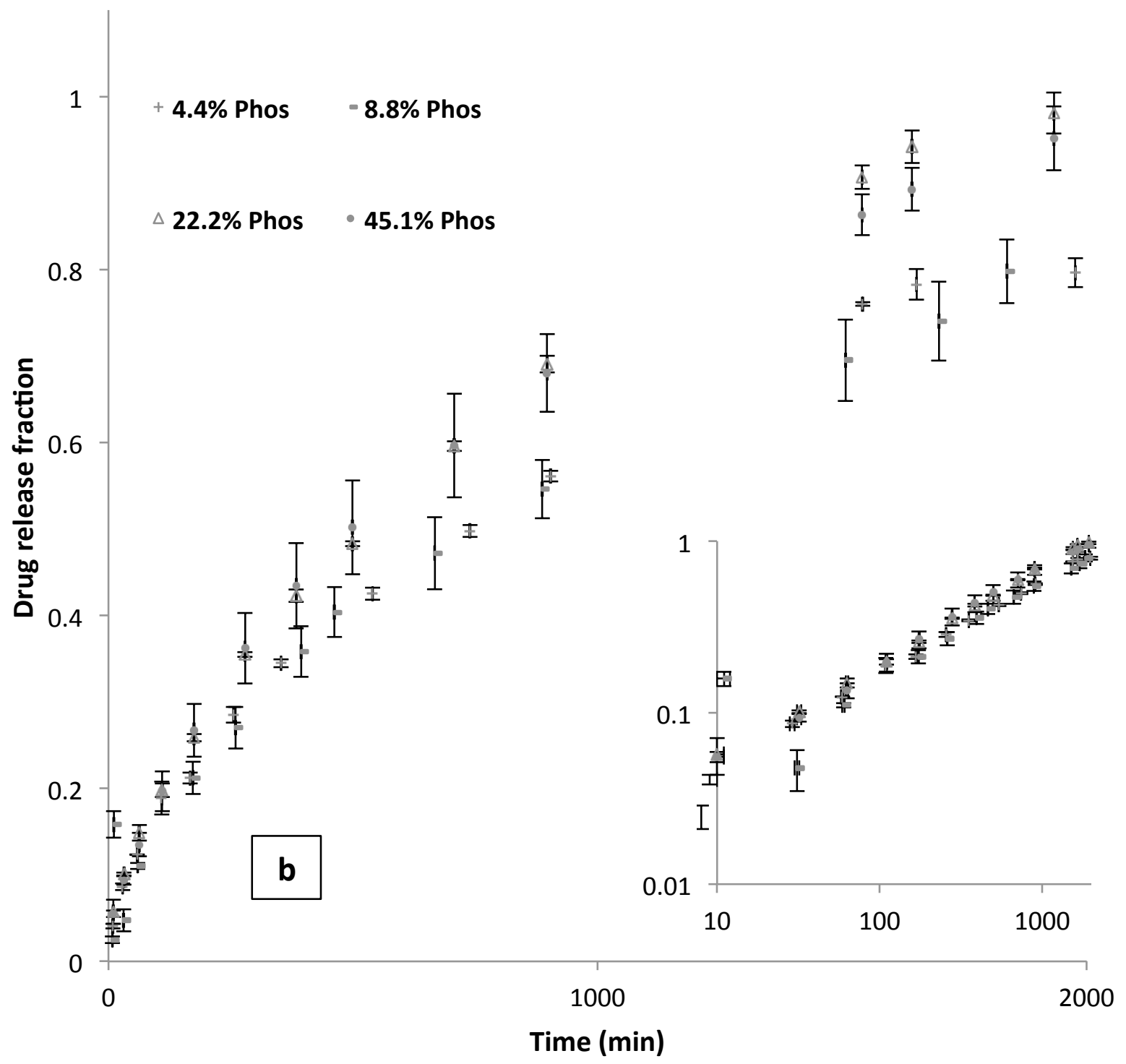


Figure 8: Dissolution of varying thickness zein/paracetamol caplet slices $(8.8 \%$ drug loading, diameter $6 \mathrm{~mm}$ ) in $\mathrm{HCl}$. Release is plotted versus time. The lines show data fits using the disk model: equation [3].

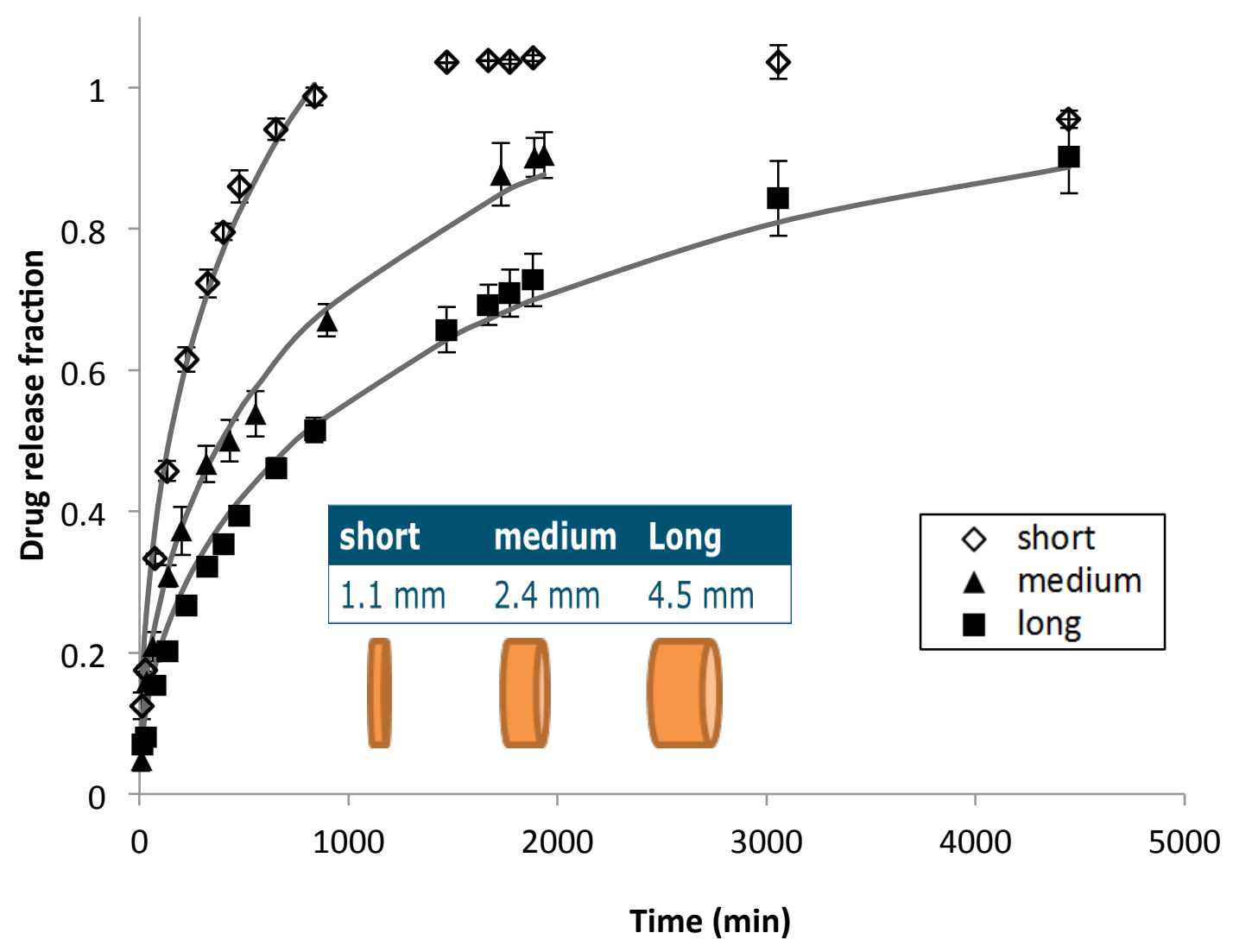

